\title{
Digital Cognitive Tests with Brazilian elderly
}

\author{
Leandro Agostini do Amaral \\ Universidade de São Paulo \\ Avenida Trabalhador São-carlense, \\ 400 - Centro \\ São Carlos, São Paulo 13566-590 \\ leandroagostini@usp.br
}

\author{
Renata Pontin de Mattos Fortes \\ Universidade de São Paulo \\ Avenida Trabalhador São-carlense, \\ 400 - Centro \\ São Carlos, São Paulo 13566-590 \\ renata@icmc.usp.br
}

\author{
Thiago Jabur Bittar \\ Universidade Federal de Goiás \\ Av. Dr. Lamartine Pinto de Avelar, \\ 1120 \\ Catalão, Goiás 75704-020 \\ thiagojabur@gmail.com
}

\begin{abstract}
Serious games are presented as a very promising mechanism in many settings, such as education and health, however its application with the elderly is little verified. In order to reproduce in digital form the results of the elderly cognitive assessments, made and validated on paper, in this study a digital cognitive test tool, with the characteristics of a serious game, was developed using the Unified Design process. As conclusion, it is suggested that it is possible to achieve results similar to those obtained by validated and paper-based tests, from a game, adapted to the needs of the elderly, in a digital cognitive test format.
\end{abstract}

\section{KEYWORDS}

Digital Cognitive Test, Serious Games, Game Accessibility

\section{INTRODUÇÃO}

O envelhecimento populacional é uma realidade mundial e no Brasil não é diferente. Segundo projeção realizada pelo sistema do IBGE [1], em novembro de 2017, a população de 50 anos, ou mais, integrava $23,34 \%$ da população brasileira, o que representa aproximadamente 48,5 milhões de habitantes. Esse percentual tende a aumentar, com previsões de que em 2030, alcance $31,2 \%$. Um grande desafio enfrentado com o envelhecimento populacional é a maior prevalência de transtornos neurocognitivos. Essa prevalência na população mundial acima de 65 anos varia entre 5 e $7 \%$, com maiores valores $(8,5 \%)$ encontrados na América Latina [2]. No Brasil, os estudos de prevalência encontraram taxas que variaram de 5,1\% [3] a 12,9\% $[4,5]$. Em número absoluto, estima-se que o Brasil seja o nono país do mundo com maior número de casos de demência, cerca de um milhão de indivíduos [2].

As demências constituem uma questão de saúde pública e trazem uma preocupação bioética: a perda da autonomia dos afetados e a responsabilidade do sistema de saúde para atendê-los [4].

O sistema biológico mais comprometido com o processo de envelhecimento é o Sistema Nervoso Central (SNC), responsável por gerar as sensações, os movimentos, funções psíquicas e

In: XVII Workshop de Ferramentas e Aplicações (WFA 2018), Salvador, Brasil. Anais do XXIV Simpósio Brasileiro de Sistemas Multimídia e Web: Workshops e Pôsteres. Porto Alegre: Sociedade Brasileira de Computação, 2018.

(C)2018 SBC - Sociedade Brasileira de Computação.

ISBN: 978-85-7669-435-9. funções biológicas internas [6]. A integridade da estrutura dos neurônios e das vias neuronais torna o SNC apto ao acúmulo de informações do presente, lembranças do passado e à formulação de novos conceitos. Assim como outros sistemas biológicos, o SNC também possui propriedades capazes de diminuir os impactos causados pelas alterações do envelhecimento [6]. O próprio envelhecimento saudável acarreta, aos idosos, alterações cognitivas [7]. As alterações mais comuns ocorrem na função executiva, atenção, memória episódica, memória de trabalho e percepção [7, 8]. A função executiva é um conjunto de habilidades, que de forma integrada, possibilita ao indivíduo direcionar comportamentos a objetivos, realizando ações voluntárias. Tais ações são auto organizadas, mediante a avaliação de sua adequação e eficiência em relação ao objetivo pretendido, de modo a eleger as estratégias mais eficientes, resolvendo assim, problemas imediatos, e/ou de médio e longo prazo. A função executiva é requerida sempre que se faz necessário formular planos de ação ou quando uma sequência de respostas apropriadas deve ser selecionada [9]; atenção é a tomada de posse, pela mente, de modo claro e vívido, de um entre os muitos objetos ou cadeias de pensamento possíveis. A focalização e a concentração da consciência fazem parte da sua essência. Ela implica o afastamento de algumas coisas de modo a que se possa lidar efetivamente com outras [10]; memória episódica é um sistema neurocognitivo que possibilita ao ser humano recordar fatos passados, podendo realizar uma "viagem no tempo", relembrando episódios de sua vida até o presente instante [11]; memória de trabalho representa um sistema de memória ultrarrápida (dura poucos segundos), que tem a capacidade de reter, por exemplo, uma sequência de 5 a 9 dígitos - o suficiente para gravar um número de telefone até efetuar a discagem, esquecendo-o logo em seguida [9] e percepção é o processo pelo qual o cérebro seleciona, organiza e interpreta estímulos, traduzindo-os em uma imagem significativa [12].

O declínio cognitivo pode comprometer a saúde do idoso, com consequente perda da capacidade funcional e autonomia, resultando em diminuição da qualidade de vida [13]. A cognição é cada vez mais reconhecida como um fator determinante para a resiliência e a homeostase corporal [14].

Como alternativa para retardar os danos à função cognitiva inerentes e que naturalmente ocorrem devido ao processo de envelhecimento, pesquisas têm sido realizadas sobre os efeitos benéficos dos jogos digitais, os quais podem revolucionar a avaliação cognitiva dos idosos, em ambientes clínicos, permitindo 
que as avaliações sejam mais frequentes, mais acessíveis e mais agradáveis [15].

Foram investigadas as principais ferramentas para teste cognitivo, atualmente existentes, que já são validadas para uso em papel. A seguir, foi proposta uma conversão de teste cognitivo para uma ferramenta em modelo digital, seguindo o processo estruturado de design de jogos, denominado Design Unificado [16].

\section{TESTE COGNITIVO DIGITAL PARA IDOSOS - CONCEITOS}

Os trabalhos estudados, com base em pesquisas publicadas e relacionadas com os tópicos desta pesquisa, possibilitaram o projeto e desenvolvimento de uma ferramenta de teste cognitivo digital voltado ao público idoso, que atendesse à demanda de proporcionar similaridade com a atividade de avaliação por meio de baterias de teste cognitivo, comumente utilizadas por profissionais da saúde.

Nesse contexto, são apresentados os conceitos de serious games e seus benefícios, da avaliação cognitiva e os domínios cognitivos, e de um conjunto de técnicas para reduzir as barreiras ao ambiente de jogos digitais, especialmente relativas à acessibilidade, pelo público idoso.

\subsection{Serious Games}

Diante dos vários domínios de conhecimento que já abordaram o tema de jogos, como: educação [17,18], saúde [19], religião [20] e política [21], e atendo-se ao segmento que detém o entretenimento como segundo plano, emerge a denominação serious games.

De modo geral, a expressão "serious games" é adotada com o significado de que os jogos dessa categoria possuem uma motivação educativa explícita, cuidadosamente pensada, e não se destinam a serem jogados exclusivamente para diversão ou entretenimento. No entanto, essa interpretação não significa que serious games não sejam, ou não devam ser, divertidos; ao contrário, a ideia é de que serious games também possuam elementos criativos e agradáveis como seus componentes.

Os serious games são projetados para terem um impacto no público-alvo, que está além do entretenimento somente [18, 22, 23]. Um dos domínios de aplicação mais importantes é o da Educação, dado seu potencial reconhecido frente à contínua necessidade de aprimoramento das técnicas e atualização de recursos educacionais [24].

Outra iniciativa mais recente para uso dos serious games é sua adoção pelo público idoso. Esse público-alvo representa uma comunidade de potenciais usuários que podem se beneficiar muito de jogos digitais. Argumenta-se que por meio de jogos digitais, é possível amenizar o declínio de alguns dos aspectos inerentes ao envelhecimento [25].

Em uma pesquisa sobre diferentes momentos e situações clínicas dos idosos, os estudos de [26] revelaram que serious games têm se mostrado muito importantes para manter e desenvolver as competências cognitivas e sociais dos idosos. Na literatura especializada, estudos apontam que serious games para idosos podem ser uma opção para melhorar os danos das funções cognitivas [27] e motoras [28], a manutenção da autoimagem e o convívio social (afetividade e solidão) [29].

Segundo [30], uma área bastante promissora para aplicação de serious games, voltados para o público idoso, é a avaliação neuropsicológica, que consiste em uma investigação minuciosa das funções cognitivas, sensoriais, motoras, emocionais e sociais da pessoa, com o intuito de se identificar algum comprometimento funcional neurológico, bem como as áreas preservadas. Podem também proporcionar outros benefícios, inerentes aos testes cognitivos computacionais [31]: melhorar a padronização da administração de testes; prover fontes mais ricas de dados sobre medidas de desempenho, como tempo de resposta, latência e variabilidade; capacitar a avaliação de um maior número de domínios; aumentar a sensibilidade; reduzir vieses culturais; aumentar a consistência entre os grupos clínicos; aumentar a eficiência e controle de qualidade para o gerenciamento de dados; ser menos estressante e fornecer maior senso de domínio e controle aos participantes.

Com base nesses trabalhos, a adoção de serious games nesta pesquisa se justifica. Na próxima subseção, são descritos os trabalhos que foram estudados, sobre a atividade de avaliação cognitiva, que tem sido realizada por profissionais da área de Saúde dos idosos.

\subsection{Avaliação Cognitiva}

$\mathrm{Na}$ avaliação cognitiva global, a grande maioria dos estudos utilizam escalas de rastreamento breves, como por exemplo o Mini Exame do Estado Mental (MEEM) [14]. O MEEM é um instrumento de rastreamento (verificação) amplamente utilizado, que apresenta escore que varia de 0 a 30, e avalia orientação temporal e espacial, memória (fixação e evocação), linguagem, atenção e cálculo. Diversos estudos de validação para determinar suas qualidades psicométricas foram realizados no Brasil, em diferentes populações [32].

Outros instrumentos para avaliação de desempenho cognitivo, que foram reportados na literatura, especialmente para uso da população brasileira, são os seguintes:

1. Lista de figuras da Bateria breve de rastreio cognitivo $(B B R C)$ : utilizando-se de figuras para avaliação de memória. Este teste consiste nas seguintes etapas: nomeação, memória incidental, memória imediata, aprendizagem, memória tardia e reconhecimento [33];

2. Teste de extensão de dígitos (ordem inversa e direta) da Wechsler Memory Scale revised: constituído por sete pares de sequências numéricas, com diferentes quantidades de dígitos, o teste é aplicado na sequência direta e inversa, sendo que durante a aplicação direta, as sequências têm de 3 a 9 números e na inversa de 2 a 8 números [34]. O teste termina após erro de duas sequências consecutivas, sendo registrada a quantidade máxima de números repetidos sem erro para cada versão;

3. Teste de nomeação de Boston: é o teste para avaliar linguagem mais utilizado no Brasil, principalmente para avaliar a capacidade de nomeação. É composto por 15 figuras, com três graus de dificuldade. A aplicação consiste em solicitar ao participante nomear os desenhos. O teste apresenta valores normativos para o Brasil [35]; 
4. Teste do Desenho do Relógio - TDR: é comumente empregado na investigação da presença de comprometimento de algumas habilidades cognitivas, como funções visuoconstrutiva e visuoespacial, representação simbólica e grafomotora, memória semântica e funções executivas. A pontuação consiste em uma escala que varia de 0 a 10 pontos. O teste encontra-se validado na população brasileira [36];

5. Teste de fluência verbal semântica: avalia diferentes domínios cognitivos como linguagem, memória semântica e função executiva. $\mathrm{O}$ examinador pede ao sujeito que fale o maior número possível de animais, no período de um minuto. A avaliação dependerá do grau de escolaridade do participante: a nota de corte para indivíduos com até oito anos de escolaridade é 9, e para indivíduos com mais de 8 anos de escolaridade, a nota de corte é 13 [37];

6. Lista de palavras do CERAD: uma lista de palavras é apresentada para o sujeito e ao final de cada apresentação deve-se recordar o maior número de palavras possível. O procedimento é repetido por três vezes. Após um período de tempo, o examinado será solicitado a evocar as palavras e em seguida reconhecer as mesmas em uma lista de 20 palavras [38];

7. Figuras geométricas do CERAD: é um subteste da bateria do CERAD [38], no qual é solicitado ao paciente desenhar uma cópia de quatro figuras geométricas simples;

8. Subtestes de semelhanças CAMDEX: constituído de quatro perguntas para avaliar a capacidade de abstração dos participantes, por exemplo, o que tem em comum uma maçã e uma banana [39];

9. Reconhecimento de emoções faciais - REF: pode ser feito pelo Penn Emotion Recognition Test, no qual fotografias de expressões faciais são apresentadas, com as seguintes emoções básicas: alegria, tristeza, raiva, medo e nojo, e faces/rostos sem emoção (neutro).

A categorização dos instrumentos estudados, separados por domínios neurocognitivos também deve ser levada em consideração de acordo com o Diagnostic and Statistical Manual of Mental Disorders (DSM-5) [40].

\subsection{Acessibilidade no Âmbito de Jogos Digitais}

Apesar do interesse crescente pelo uso de jogos digitais em diferentes áreas, muitas pessoas, dentre as quais os idosos, acabam por serem privadas de utilizá-los, seja por algum declínio, restrição visual, motora, auditiva ou cognitiva [41-43].

Para considerar as limitações dos indivíduos que podem ser atingidos pela falta de acessibilidade, utiliza-se, em geral, a classificação definida pela World Health Organization's (WHO) em International Classification of Impairments, Disabilities, and Handicaps (ICIDH), que abrange os seguintes comprometimentos: visual, que é a consequência de um certo grau de perda de visão; auditivo, o qual refere-se à perda parcial ou total da habilidade de ouvir, por um ou pelos dois ouvidos; motor, que é a perda ou limitação da função de controle muscular, de movimento ou limitação da mobilidade; cognitivo, que representa uma enfermidade mental ou psicológica, que vai desde um retardo desenvolvido durante a infância, até Alzheimer ou senilidade, como resultado do processo de envelhecimento.

Conforme a popularidade da tecnologia aumenta, intensificam-se os esforços para compreender aqueles atingidos por esses comprometimentos, bem como tornar a tecnologia acessível ao maior número de pessoas. Um resultado desse direcionamento é referência mundial e consiste na Web Accessibility Initiative (WAI), que elaborou o documento Web Content Accessibility Guidelines (WCAG), desenvolvido a partir do processo [44] do World Wide Web Consortium (W3C);

As diretrizes WCAG orientam a produção de conteúdo (textos, imagens, formulários, sons) para a web, que seja acessível às pessoas com a diversidade de tipos de deficiência.

Com a realidade do aumento da popularidade dos jogos digitais, o enfoque de trabalhos e pesquisas no sentido de prover acessibilidade a esse tipo de sistemas interativos também deve aumentar, porém ainda são poucos os esforços neste sentido [45]. Não há diretrizes oficiais, padrões ou iniciativas globais, comparáveis às WCAG, no domínio de jogos digitais, tampouco ações governamentais ou legislativas relacionadas, entretanto, existem duas intervenções no sentido de obter esse conjunto de diretrizes: uma publicada pelo Special Interest Group (SIG), da Independent Game Developers Association (IGDA), que propôs 19 diretrizes de acessibilidade, em 2004, e atualizadas em 2010, obtidas de um experimento com 20 jogos, priorizando os usuários com deficiência visual e outra, da organização norueguesa MediaLT, que também em 2004, publicou um conjunto de 34 diretrizes de acessibilidade.

Mais recentemente, em 2012, visando produzir uma referência objetiva aos desenvolvedores de jogos digitais, e incluir o maior número de pessoas possível, considerando as diferentes necessidades apresentadas por pessoas com deficiências, um esforço colaborativo entre produtores, especialistas e acadêmicos foi realizado, surgindo as diretrizes de acessibilidade para jogos. Paralelamente à obtenção de diretrizes de acessibilidade para jogos digitais, formalizadas internacionalmente, evidencia-se o avanço da qualidade dos jogos, a partir da criação de um processo estruturado de design de jogos, denominado Design Unificado (DU) [16]. O DU inclui como uma de suas etapas, a avaliação de acessibilidade técnica, que pode verificar o uso com base nas diretrizes, mas também recorrer à participação de especialistas e usuários finais.

\section{MÉTODO E RESULTADOS}

Este estudo é de natureza transversal de levantamento de dados, descritivo e analítico, a partir de uma investigação qualiquantitativa. A pesquisa foi realizada na cidade de São Carlos, situada no Estado de São Paulo, Brasil.

Para o desenvolvimento do teste cognitivo digital, considerou-se o processo DU na fase de análise e o Integrated Development Environment (IDE) Stencyl foi utilizado para desenvolvimento.

$\mathrm{O}$ tablet foi escolhido como dispositivo final, considerando a eficácia e assertividade no uso pela população idosa, que, com dificuldades psicomotoras e audiovisuais, encontra, nesse dispositivo, uma ferramenta mais acessível ao ambiente virtual, vencendo algumas barreiras relativas às dificuldades psicomotoras.

O estudo, para identificar e projetar a interface mais adequada ao público idoso, considerou questões de acessibilidade desde seu planejamento, por meio do processo DU e pesquisas sobre como avaliar a acessibilidade em jogos digitais, especialmente para esse público.

Um exemplo de decisão tomada durante o desenvolvimento do protótipo, além da observação das diretrizes de acessibilidade, foi 
a escolha da paleta de cores, realizada respeitando-se a possibilidade de atender indivíduos que apresentassem daltonismo, mais especificamente Tritanopia, Protanopia e Deuteranopia. A ferramenta Color Oracle foi utilizada para averiguar a paleta nas três situações e então foram eliminadas cores que se aproximavam umas das outras, tornando-as quase indistinguíveis. Ainda assim foi necessário o devido cuidado ao confeccionar os assets do jogo, isto é, feedbacks e objetivos não podem contar apenas com a cor para diferenciar objetos; portanto, sons e formas também foram utilizados.

$\mathrm{Na}$ bateria de testes cognitivos, foram apurados nove aspectos, a saber: 01 - Percepção Visual, Memória Incidental e Memória Imediata, 02 - Praxia, 03 - Abstração, 04 - Percepção Visual, Memória Incidental e Memória Imediata, 05 - Teste de Extensão de Dígitos (Direto), 06 - Teste de Extensão de Dígitos (Inverso), 07 - Atenção, 08 - Teste de Fluência Verbal e 09 Reconhecimento Facial. Esses aspectos foram apurados e serviram para analisar uma possível correlação, que demonstrasse a portabilidade da avaliação convencional (em papel), para o meio digital, sem que houvesse uma alteração significativa no contexto. Nas Figuras 1, 2, 3, apresentadas a seguir, é possível verificar algumas das telas da ferramenta, com elementos de fácil utilização pelo tamanho e cores para a execução dos testes cognitivos.

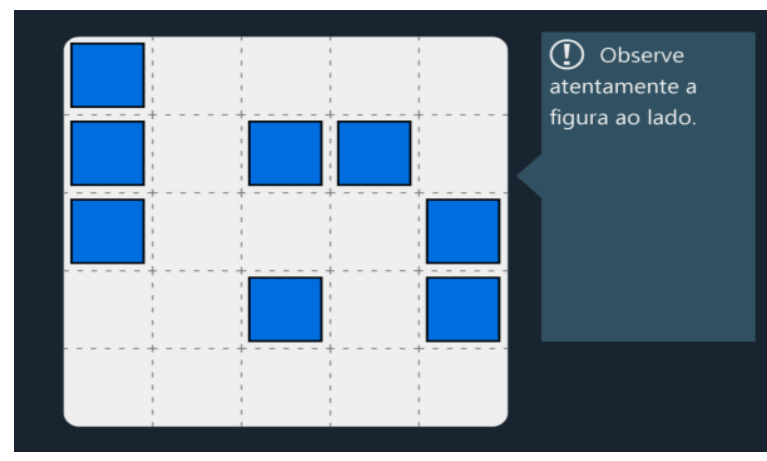

Figura 1: Tela para obter instruções sobre como identificar a diferença na matriz.

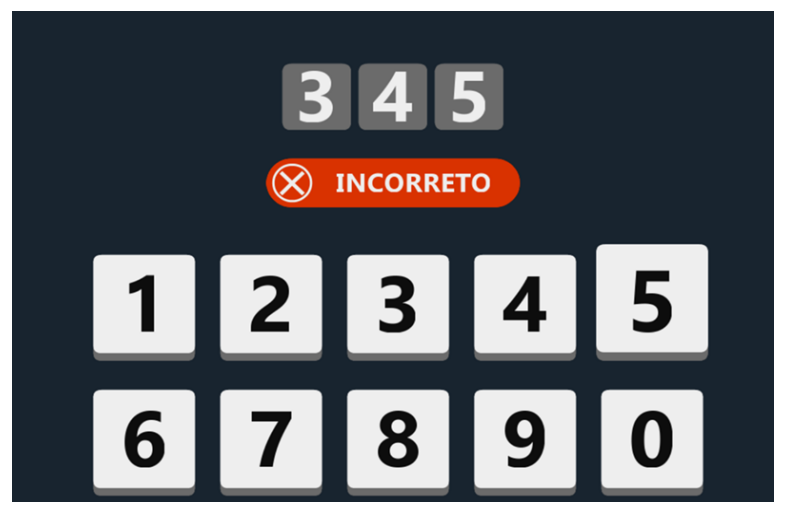

Figura 2: Tela para relatar falhas ao lembrar uma sequência de números.

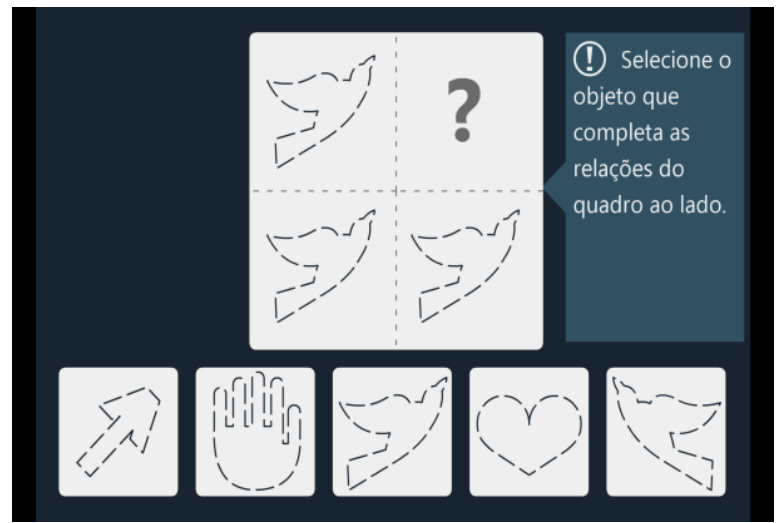

Teste baseado no Beta III.

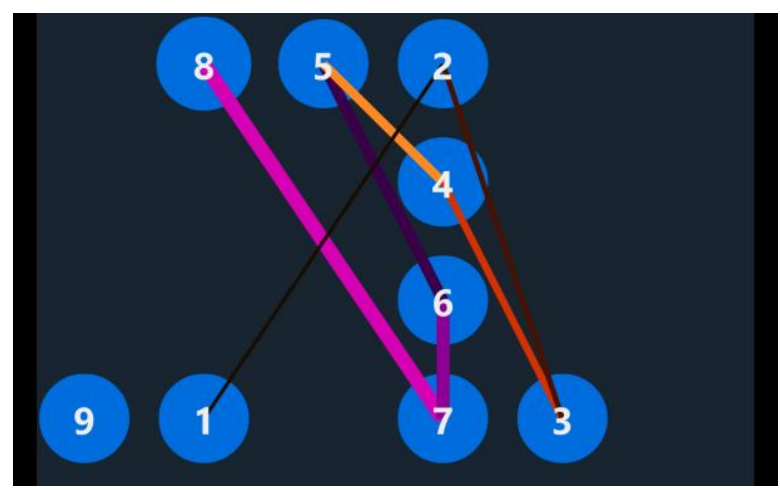

Figura 3: Teste baseado no Trail Making Test.

Para cada um dos testes, tem-se medidas (atribuídas a variáveis), que foram capturadas durante o processo de interação do idoso. Essas medidas foram utilizadas na fase de análise da convergência, com os testes validados em papel. O objetivo principal no uso dessas variáveis é representar o resultado de cada teste e possibilitar análise computacional, sem a percepção humana, a fim de inibir vieses por parte do aplicador. Um vídeo demonstrando a execução do teste cognitivo digital, resultado desse estudo, pode ser visto em: http://leandroagt.com.br/video.mp4.

\section{CONCLUSÕES}

Os serious games têm se mostrado relevantes quando utilizados em diversos tipos de aplicações, como é o caso de apoiar atividades no campo da saúde. Nesta pesquisa, foi investigada sua adoção considerando procedimentos de avaliação cognitiva com o público idoso do Brasil. De fato, as tecnologias digitais se tornam cada vez mais uma realidade para o mundo atual, e os novos idosos já têm sido introduzidos a elas, muitas vezes, desde seu próprio ambiente familiar ou de trabalho.

Assim, esta ferramenta que interliga o potencial de jogos, por meio de serious games, à demanda da sociedade atual, cujo contingente populacional se expande rapidamente, se apresenta como um quesito mais que natural. Esse contexto motivou a pesquisa descrita, a qual sugere que é possível atingir resultados 
semelhantes, a partir de um jogo, adaptado às necessidades do idoso, em formato de teste cognitivo digital, aos obtidos pelos testes baseados em papel, já validados e comumente utilizados por profissionais da saúde. Com esse resultado, poder-se-á utilizar o teste cognitivo digital para o âmbito de pesquisa acadêmica, bem como na identificação de perfis dos usuários para um projeto de produto em andamento, que é o ambiente de treinamento cognitivo, por meio de jogos digitais, direcionados aos declínios identificados no jogador.

\section{AGRADECIMENTOS}

O presente trabalho foi realizado com apoio da Coordenação de Aperfeiçoamento de Pessoal de Nível Superior - Brasil (CAPES) Código de Financiamento 001 e pela FAPESP - projeto número 2016/01009-0.

\section{REFERÊNCIAS}

[1] IBGE. Projeção da população, http://www.ibge.gov.br/apps/populacao/projecao/

[2] Prince M., Bryce R., Albanese E., Wimo A., Ribeiro W., Ferri C.P.: The global prevalence of dementia: a systematic review and meta-analysis Alzheimers. Dement., 9, pp. 63-75. e2 (2013)

[3] Scazufca M., Menezes P.R., Vallada H.P., Crepaldi A.L., Pastor-Valero M., Coutinho L.M.S., Di Rienzo V.D., Almeida O.P.: High prevalence of dementia among older adults from poor socioeconomic backgrounds in São Paulo, Brazil Int. Psychogeriatr., 20, pp. 394-405 (2008)

[4] Burlá C., Camarano A.A., Kanso S., Fernandes D., Nunes R.: Panorama prospectivo das demências no Brasil: um enfoque demográfico Cien. Saude Colet., 18, pp. 2949-2956 (2013)

[5] Bottino C.M.C., Azevedo D., Jr., Tatsch M., Hototian S.R., Moscoso M.A., Folquitto J., Scalco A.Z., Bazzarella M.C., Lopes M.A., Litvoc J.: Estimate of Dementia Prevalence in a Community Sample from São Paulo, Brazil Dement. Geriatr. Cogn. Disord., 26, pp. 291-299 (2008)

[6] Py L., de Freitas E.V., do Gorzoni M.L.: Tratado de geriatria e gerontologia, Grupo Gen - Guanabara Koogan, (2011)

[7] Ferreira D., Molina Y., Machado A., Westman E., Wahlund L.-O., Nieto A., Correia R., Junqué C., Díaz-Flores L., Barroso J.: Cognitive decline is mediated by gray matter changes during middle age Neurobiol. Aging, 35, pp. 1086-1094 (2014)

[8] Mishra J., Rolle C., Gazzaley A.: Neural plasticity underlying visual perceptual learning in aging Brain Res., 1612, pp. 140-151 (2015)

[9] Mourão Junior C.A., Junior C.A.M., Melo L.B.R.: Integração de três conceitos: função executiva, memória de trabalho e aprendizado Psicologia: Teoria e Pesquisa, 27, pp. 309-314 (2011)

[10] Kandel E.R., Rubino R.: Em busca da memória: o nascimento de uma nova ciência da mente, (2009)

[11] Tulving E.: Episodic Memory: From Mind to Brain Annu. Rev. Psychol., 53, pp. $1-25(2002)$

[12] Lamb C.W., Hair J.F., McDaniel C.: Marketing, South Western Educationa Publishing, (2004)

[13] Grden C.R.B., Barreto M.F.C., Sousa J.A.V. de, Chuertniek J.A., Reche P.M., Borges P.K. de O.: Association between physical frailty and cognitive scores in older adults Rev Rene, 16, pp. 391-397 (2015)

[14] Canevelli M., Cesari M., van Kan G.A.: Frailty and cognitive decline: how do they relate? Curr. Opin. Clin. Nutr. Metab. Care, 18, pp. 43-50 (2015)

[15] Tong T., Chignell M., Tierney M.C., Lee J.: A Serious Game for Clinical Assessment of Cognitive Status: Validation Study JMIR Serious Games, 4, pp. e7 (2016)

[16] Grammenos D., Savidis A., Stephanidis C.: Designing Universally Accessible Games Human Factors and Ergonomics. pp. 1-12 (2009)

[17] Gee J.P.: What video games have to teach us about learning and literacy Computers in Entertainment, 1, pp. 20 (2003)

[18] Kebritchi M., Hirumi A.: Examining the pedagogical foundations of modern educational computer games Comput. Educ., 51, pp. 1729-1743 (2008)

[19] Lieberman D.A.: Interactive Video Games for Health Promotion: Effects on Knowledge, Self-Efficacy, Social Support, and Health Health Promotion and Interactive Technology: Theoretical Applications and Future Directions. pp. 103-120 (1997)

[20] NBC. Give me that online religion, http://www.nbcnews.com/id/18789168/ns/nbc_nightly_news_with_brian_willia $\mathrm{ms} / \mathrm{t} /$ give-me-online-religion/
[21] Delhi J.P. in: Tiny island nation opens the first real embassy in virtual world, https://www.thetimes.co.uk/article/tiny-island-nation-opens-the-first-realembassy-in-virtual-world-c3phsx2n2r0

[22] Michael D.R., Chen S.: Serious Games: Games that Educate, Train and Inform, Course Technology, (2006)

[23] Greitzer F.L., Kuchar O.A., Huston K.: Cognitive science implications for enhancing training effectiveness in a serious gaming context. ACM J. Educ. Resour. Comput., 7, pp. 2-es (2007)

[24] Frederik D.G., Peter M., Van Looy J.: Uncharted waters? Proceedings of the 3 rd International Conference on Fun and Games - Fun and Games '10 (2010)

[25] Chen S.-T., Huang Y.-G.L., Chiang I.-T.: Using Somatosensory Video Games to Promote Quality of Life for the Elderly with Disabilities 2012 IEEE Fourth International Conference On Digital Game And Intelligent Toy Enhanced Learning (2012)

[26] Programa de estimulação cognitiva em idosos institucionalizados, http://www.psicologia.pt/artigos/ver_artigo.php?codigo=a0623

[27] Torres A.C.S.: Cognitive effects of video games on old people Int. J. Disabil. Hum. Dev., 10, (2011)

[28] Wiemeyer J., Kliem A.: Serious games in prevention and rehabilitation-a new panacea for elderly people? Eur. Rev. Aging Phys. Act., 9, pp. 41-50 (2011)

[29] Jung Y., Li K.J., Janissa N.S., Gladys W.L.C., Lee K.M.: Games for a better life Proceedings of the Sixth Australasian Conference on Interactive Entertainment - IE'09 (2009)

[30] Valladares-Rodríguez S., Pérez-Rodríguez R., Anido-Rifón L., FernándezIglesias M.: Trends on the application of serious games to neuropsychological evaluation: A scoping review J. Biomed. Inform., 64, pp. 296-319 (2016)

[31] Espeland M.A., Katula J.A., Rushing J., Kramer A.F., Jennings J.M., Sink K.M., Nadkarni N.K., Reid K.F., Castro C.M., Church T., Kerwin D.R., Williamson J.D., Marottoli R.A., Rushing S., Marsiske M., Rapp S.R., LIFE Study Group: Performance of a computer-based assessment of cognitive function measures in two cohorts of seniors Int. J. Geriatr. Psychiatry, 28, pp. 1239-1250 (2013)

[32] Brucki S.M.D., Nitrini R., Caramelli P., Bertolucci P.H.F., Okamoto I.H.: [Suggestions for utilization of the mini-mental state examination in Brazil] Arq Neuropsiquiatr., 61, pp. 777-781 (2003)

[33] Nitrini R., Lefèvre B.H., Mathias S.C., Caramelli P., Carrilho P.E., Sauaia N., Massad E., Takiguti C., Da Silva I.O., Porto C.S.: [Neuropsychological tests of simple application for diagnosing dementia] Arq. Neuropsiquiatr., 52, pp. $457-$ 465 (1994)

[34] Dickson A.L.: Book Review: Lezak, Muriel Deutsch, (1983) Neuropsychological assessment (2nd ed.). New York: Oxford University Press J. Psychoeduc. Assess., 4, pp. 91-92 (1986)

[35] Kaplan E., Goodglass H., Weintraub S.: Boston Naming Test, http://dx.doi.org/10.1037/t27208-000

[36] Fuzikawa C., Lima-Costa M.F., Uchoa E., Barreto S.M., Shulman K., Bambuí Health and Ageing Study: A population-based study on the intra and inter-rater reliability of the clock drawing test in Brazil: the Bambuí Health and Ageing Study Int. J. Geriatr. Psychiatry, 18, pp. 450-456 (2003)

[37] Brucki S.M.D., Rocha M.S.G.: Category fluency test: effects of age, gender and education on total scores, clustering and switching in Brazilian Portuguesespeaking subjects Braz. J. Med. Biol. Res., 37, pp. 1771-1777 (2004)

[38] Moms J.C., Heyman A., Mohs R.C., Hughes J.P., van Belle G., Fillenbaum G., Mellits E.D., Clark C.: The Consortium to Establish a Registry for Alzheimer's Disease (CERAD). Part I. Clinical and neuropsychological assesment of Alzheimer's disease Neurology, 39, pp. 1159-1159 (1989)

[39] Roth M., Huppert F.A., Tym E., Mountjoy C.Q., Diffident-Brown A., Shoesmith D.J.: The Cambridge Examination for Mental Disorders of the Elderly: CAMDEX, Cambridge University Press, (1988)

[40] DSM-5, https://www.psychiatry.org/psychiatrists/practice/dsm

[41] Atkinson M.T., Gucukoglu S., Machin C.H.C., Lawrence A.E.: Making the Mainstream Accessible: What's in a Game? Lecture Notes in Computer Science. pp. 380-387 (2006)

[42] Grammenos D.: Game over Proceeding of the twenty-sixth annual CHI conference on Human factors in computing systems - CHI'08 (2008)

[43] Bierre K., Chetwynd J., Ellis B., Michelle D., Ludi S., Westin T.: Game Not Over: Accessibility Issues in Video Games Proc. of the 3rd International Conference on Universal Access in Human-Computer Interaction (2005)

[44] How WAI Develops Accessibility Guidelines through the W3C Process: Milestones and Opportunities to Contribute $\circ$ Web Accessibility Initiative W3C, https://www.w3.org/WAI/intro/w3c-process.php

[45] Yuan B., Folmer E., Harris F.C.: Game accessibility: a survey Universal Access in the Information Society, 10, pp. 81-100 (2010) 